\title{
DEVELOPMENT AND VALIDATION OF NEW ANALYTICAL METHOD FOR THE DETERMINATION OF PARTICLE SIZE DISTRIBUTION OF TADALAFIL DRUG SUBSTANCE USING LASER PARTICLE ANALYZER
}

\author{
MINGJIN XUN ${ }^{1,2 *}$, HUI GUO ${ }^{1,2}$, WENFANG LI ${ }^{1,2}$, GUIMIN ZHANG $^{1,2 *}$, \\ ZHONG FENG ${ }^{1,2 *}$, and MENGHUA WANG ${ }^{1,2}$
}

\author{
${ }^{1}$ National Engineering and Technology Research Center of Chirality Pharmaceutical, \\ Lunan Pharmaceutical Group Co., Ltd., Liny City Shandong \\ ${ }^{2}$ International Pharmaceutical Engineering Lab in Shandong Province, \\ Shandong New Time Pharmaceutical Co., Ltd., Feixian, Shandong China
}

\begin{abstract}
The Malvern Mastersizer 3000 laser particle size analyzer and HydroMV wet sampler were used, and the parameters are set as follows: sample refractive index 1.52 , funnel width $2.0 \mathrm{~mm}$, dispersion air pressure 4.0 bar, ultrasonic intensity $50 \%$, ultrasonic time $30 \mathrm{~s}$, stirring speed $1600 \mathrm{rpm}$, laser obscuration $8 \% \sim 15 \%$. The mean values of particle size distribution $\mathrm{d}(0.1), \mathrm{d}(0.5)$, and $\mathrm{d}(0.9)$ of 3 batches of tadalafil drug substance were $0.819 \mu \mathrm{m}, 2.79 \mu \mathrm{m}$, and $8.44 \mu \mathrm{m}$, respectively. The measured results were basically consistent with the microscopic method. The method is simple, accurate and precise, and suitable for the particle size determination of tadalafil drug substance.
\end{abstract}

Keywords: laser light scattering method, tadalafil, particle size distribution

Tadalafil is the first-line treatment option for erectile dysfunction (ED) in males and is also used for pulmonary arterial hypertension (PAH), a debilitating chronic disease of the small pulmonary arteries (1-3). Tadalafil is a water-insoluble drug, classified as a BCS class II drug. The manufacturability of tablets, the dissolution rate, which is proportional to the surface area of drugs, is largely dependent on the particle size distribution of drug particles (4). Consequently, the particle size has a profound influence on almost every step in tablet manufacturing, including mixing (5), granulation (6), compression (7), and coating (8). Therefore, the particle size distribution of tadalafil is one of the important control indexes in the process of preparation and pharmaceutical production. Hence, the development of a reliable and robust method for the determination of particle size of any active pharmaceutical ingredient is critical.

A literature survey was done for the Particle size distribution method for tadalafil and found that the particle size determination method is not available. Therefore, this study was carried out to develop a method to determine the particle size distribution of tadalafil by Particle size analyzer, and further validation of the method was carried out.
There are many different techniques available for particle size analysis like optical microscopy, laser diffraction, sieving, light scattering, sedimentation, porosimetry, etc. (9). Laser diffraction is a widely used particle sizing technique for materials ranging from hundreds of nanometers up to several millimeters in size (10). The angular scattering intensity data is then analyzed to calculate the size of the particles responsible for creating the scattering pattern, using the Mie theory of light scattering. The particle size is reported as a volume equivalent sphere diameter. For particles of $<50 \mu \mathrm{m}$, the Mie theory light intensity model is more accurate.

Malvern Mastersizer 3000 laser diffraction particle size analyzer consistently delivers accurate, robust, reliable data that informs critical decision-making throughout $\mathrm{R} \& \mathrm{D}$ and manufacturing processes.

\section{EXPERIMENTAL}

\section{Materials}

Tadalafil drug substance were purchased from Shandong Topharman Pharmaceutical Co., Ltd.; Tween-80 were purchased from China Sinopharm Group Co. Ltd.; water is purified water.

\footnotetext{
* Corresponding author: e-mail: zhiyao200609@163.com (M.X.); lunanzhangguimin@163.com (G.Z.); fengzhong22@163.com (Z.F.)
} 


\section{Instrument method settings}

Sample refractive index 1.52; Sample absorption rate 0.1 ; Dispersion medium (water) refractive index 1.33; Analysis mode general mode; Sensitivity common; Funnel width $2.0 \mathrm{~mm}$; Dispersion air pressure 4.0 bar; Ultrasonic intensity $50 \%$;Ultrasonic time 30S; Stirring speed 1600 rpm; Injection speed 35\%; Number of measurements 6; Measurement time: $10 \mathrm{~s}$; Background time $10 \mathrm{~s}$; Shading degree of $8 \% \sim 15 \%$. The experimental data were collected and analyzed using MasterSizer 3000 Workstation.

\section{Preparation of test solution}

Weigh $200 \mathrm{mg}$ of tadalafil drug substance, add $20 \mathrm{mg}$ of Tween 80 , stir to prepare a uniform paste; add $30 \mathrm{~mL}$ of purified water, ultrasound for $2 \mathrm{~min}$ until there are no sample particles on the surface of the liquid level, and obtain an evenly dispersed suspension.

\section{Measurement method}

According to the standard operating procedure for Mastersizer 3000 laser particle sizer, set the instrument parameters according to section " 3.1 ". After background measurement, slowly add the sample prepared under the section "3.2.1", until the obscuration state bar enters the green part, and then start determination.

\section{RESULTS (11-12)}

\section{Selection of test conditions (13)}

\section{Selection of dispersion medium and surfactant}

According to the physical and chemical properties published in the specifications of the product marketed abroad, tadalafil is freely soluble in dimethyl sulfoxide, slightly soluble in dichloromethane, and almost insoluble in water. So purified water is the first choice as the dispersion medium. To an appropriate amount of tadalafil drug substance, purified water was added and fully stirred. It was observed that tadalafil powder did not completely disperse in purified water; a small part is suspended in purified water and there is an agglomeration phenomenon. Most of the tadalafil is in a floating state, so it is necessary to add surfactant. The addition of an appropriate amount of Tween 80 can reduce its surface tension and make the sample disperse well in the medium. Therefore to an appropriate amount of tadalafil drug substance, three different quantities of Tween 80 were used to prepare the sample and set the instrument parameters. The determination was done 6 times for each sample according to the above method, and the mean $d(0.1), d(0.5), d(0.9)$ values, and weighted residual error are shown in Table 1.

The results showed that when the amount of Tween 80 was $10 \mathrm{mg}$, the drug substance could not be fully mixed in the medium, which made some drug substances agglomerate and float on the surface of purified water. As a result, the large particles were not easy to enter the detection cell, resulting in small detection results. On the other hand, when the amount dosage of Tween 80 was $50 \mathrm{mg}$, the foam was produced in the dispersion device during the detection process, and the light intensity of the system was unstable, resulting in increased measurement error. For a comprehensive comparison, the quantity of Tween 80 was selected as $20 \mathrm{mg}$.

\section{Selection of refractive index}

An appropriate amount of tadalafil drug substance was used to prepare the sample and set the instrument parameters. Different refractive indices were set and the measurement was repeated 6 times for each sample.The particle size $d(0.1), d(0.5), d(0.9)$, and weighted residual error of the 6 measurements were determined and the mean value and RSD results are shown in Table 2.

Table 1. Investigation results with different amount of Tween $80(n=6)$.

\begin{tabular}{|c|c|c|c|c|}
\hline Tween $80 / \mathrm{mg}$ & $d(0.1) / \mu \mathrm{m}$ & $d(0.5) / \mu \mathrm{m}$ & $d(0.9) / \mu \mathrm{m}$ & Mean residual /\% \\
\hline 10 & $0.827(1.49)$ & $2.33(4.00)$ & $7.09(5.68)$ & 3.31 \\
\hline 20 & $0.826(1.04)$ & $2.77(2.83)$ & $8.37(3.75)$ & 1.12 \\
\hline 50 & $0.827(1.59)$ & $2.75(5.27)$ & $8.65(8.03)$ & 6.55 \\
\hline
\end{tabular}

Table 2. Investigation results with different refractive index $(n=6)$.

\begin{tabular}{|c|c|c|c|c|}
\hline Refractive Index & $d(0.1) / \mu \mathrm{m}$ & $d(0.5) / \mu \mathrm{m}$ & $d(0.9) / \mu \mathrm{m}$ & Mean residual $/ \%$ \\
\hline 1.50 & $0.800(1.65)$ & $2.70(4.69)$ & $8.51(3.22)$ & 2.25 \\
\hline 1.51 & $0.819(1.37)$ & $2.77(4.68)$ & $8.76(4.17)$ & 3.36 \\
\hline 1.52 & $0.799(1.36)$ & $2.60(2.87)$ & $8.29(1.54)$ & 0.66 \\
\hline 1.53 & $0.826(1.61)$ & $2.76(4.39)$ & $8.47(5.04)$ & 3.78 \\
\hline
\end{tabular}


It can be seen from Table 2 that the particle size distributions at different refractive indices are in good agreement. When the refractive index is 1.52 , the residual value of the sample is minimum, so this refractive index is selected as the best measurement condition.

\section{Selection of obscuration}

According to the Chinese Pharmacopoeia, the sample size required for wet method measurement should reach $8 \% \sim 20 \%$ of the obscuration of the detector. In this experiment, the obscuration of $8 \%$, $12 \%, 15 \%$, and $20 \%$ was investigated. Table 3 shows the mean values of $d(0.1), d(0.5), d(0.9)$, and weighted residual error of the 6 readings at the selected obscuration.

The results showed that when the obscuration was $20 \%$, there were many sample particles, which caused the multiple diffractions of laser, and the measured values were quite different. However, when the obscuration was $8 \% \sim 15 \%$, the measured values of particle size were close, and the measured differences were small, so the range of obscuration was determined to be $8 \% \sim 15 \%$.

\section{Selection of dispersion air pressure}

An appropriate amount of samples were used to prepare the samples and the dispersion pressure was set as 1.5 bar, 2.0 bar, 3.0 bar, 4.0 bar, and 5.0 bar. Repeated measurements of 6 times for each sample were determined and the mean $d(0.1), d(0.5), d(0.9)$ values, and weighted residual error were calculated. The determination results are shown in Table 4.

When the disperse gas pressure is less than 3.0 bar, the samples are not uniformly dispersed and agglomerate due to the low air pressure, resulting in larger particle size measurement results. When the pressure of dispersed gas is 4.0 bar and $5.0 \mathrm{bar}$, the sample can be uniformly dispersed in purified water, and the measurement results are similar. Since the RSD value and residual error of the measurement results are the smallest when the dispersion gas pressure is 4.0 bar, the dispersion gas pressure is finally selected as 4.0 bar.

\section{Selection of ultrasonic intensity}

Taking an appropriate amount of Tadalafil drug substance, the samples were prepared and the instrument parameters were set. The ultrasonic intensity was set at $0,30 \%, 40 \%$, and $50 \%$, respectively, and the measurements were repeated 6 times for each sample.The $d(0.1), d(0.5), d(0.9)$ data and weighted residual error of the 6 measurements were determined and then the mean values and RSD were calculated. The determination results are shown in Table 5 .

Table 3. Investigation results with different obscuration $(n=6)$.

\begin{tabular}{|c|c|c|c|c|}
\hline Obscuration $/ \%$ & $d(0.1) / \mu \mathrm{m}$ & $d(0.5) / \mu \mathrm{m}$ & $d(0.9) / \mu \mathrm{m}$ & Mean residual $/ \%$ \\
\hline 8 & $0.812(1.78)$ & $2.81(2.82)$ & $8.63(3.92)$ & 1.23 \\
\hline 12 & $0.817(1.51)$ & $2.79(2.26)$ & $8.44(3.64)$ & 1.17 \\
\hline 15 & $0.823(1.09)$ & $2.78(2.16)$ & $8.54(2.43)$ & 0.98 \\
\hline 20 & $0.805(3.79)$ & $2.72(6.96)$ & $8.30(6.23)$ & 4.21 \\
\hline
\end{tabular}

Table 4. Investigation results with different air pressure $(n=6)$.

\begin{tabular}{|c|c|c|c|c|}
\hline Air pressure $/$ bar & $d(0.1) / \mu \mathrm{m}$ & $d(0.5) / \mu \mathrm{m}$ & $d(0.9) / \mu \mathrm{m}$ & Mean residual $/ \%$ \\
\hline 1.5 & $1.20(7.27)$ & $3.31(3.02)$ & $12.2(8.21)$ & 6.73 \\
\hline 2.0 & $1.10(4.88)$ & $3.23(2.40)$ & $11.3(7.10)$ & 5.88 \\
\hline 3.0 & $1.01(3.84)$ & $3.03(2.89)$ & $10.1(5.59)$ & 3.46 \\
\hline 4.0 & $0.826(0.75)$ & $2.80(1.90)$ & $8.68(2.13)$ & 0.87 \\
\hline 5.0 & $0.825(1.84)$ & $2.78(3.40)$ & $8.54(5.57)$ & 2.54 \\
\hline
\end{tabular}

Table 5. Investigation results with different ultrasonic intensity $(n=6)$.

\begin{tabular}{|c|c|c|c|c|}
\hline Ultrasonic Intensities/\% & $d(0.1) / \mu \mathrm{m}$ & $d(0.5) / \mu \mathrm{m}$ & $d(0.9) / \mu \mathrm{m}$ & Mean residual/\% \\
\hline 0 & $1.18(1.31)$ & $3.35(3.16)$ & $10.2(4.51)$ & 6.43 \\
\hline 30 & $1.10(1.29)$ & $3.15(3.05)$ & $9.81(4.71)$ & 5.05 \\
\hline 40 & $1.04(1.31)$ & $3.15(2.11)$ & $9.32(3.43)$ & 3.89 \\
\hline 50 & $0.813(1.13)$ & $2.74(2.10)$ & $8.07(3.20)$ & 0.57 \\
\hline
\end{tabular}


When the ultrasonic intensity was less than $40 \%$, the samples could not be completely dispersed in the purified water and agglomeration occurred, which made the measurement results larger. When the ultrasonic intensity is $50 \%$, the samples are uniformly dispersed in the purified water and the particle size measurement results are relatively stable. So the ultrasonic intensity of the sample is determined to be $50 \%$.

\section{Selection of ultrasonic time}

The particle size measurement results were determined when the ultrasonic time was set at 30s, $60 \mathrm{~s}$, and $120 \mathrm{~s}$, respectively. The mean $d(0.1), d(0.5)$, $d(0.9)$ values and weighted residual error were determined and are shown in Table 6.

When the ultrasonic time reaches over $60 \mathrm{~s}$, the particle sizes relatively remain unchanged. However, too long ultrasonic time should be avoided because of the possibility of particle fragmentation, so the ultrasonic time was maintained as $60 \mathrm{~s}$.

\section{Investigation of stirring speed}

Following the samples preparation as previously explained and setting the instrument parameters, the particle size determination at stirring speeds of $1200 \mathrm{rpm}, 1400 \mathrm{rpm}$, $1600 \mathrm{rpm}, 2000 \mathrm{rpm}$, and $2500 \mathrm{rpm}$ was investigated then the $d(0.1), d(0.5), d(0.9)$ values and weighted residual error of 6 measurements were determined. The mean values and RSD were calculated and the determined results are shown in Table 7.

When the stirring speed is less than $1400 \mathrm{rpm}$, large particles sink at the bottom of the injection cup and cannot enter the detection system, which makes the measurement results small particularly d (90). When the stirring speed is $1600 \mathrm{rpm}, 2000 \mathrm{rpm}$, and $2500 \mathrm{rpm}$, the particle size measurement results reach the platform value, the particle size remains the same. However, when the stirring speed is too fast, there may be bubbles, so the stirring speed is determined to be $1600 \mathrm{rpm}$.

\section{Investigation of injection speed}

Take an appropriate amount of samples, prepare the samples and set the instrument parameters, sample injection rates were set at $35 \%, 40 \%, 45 \%$, and $50 \%$, repeat the measurement 6 times for each sample, count the $d(0.1), d(0.5), d(0.9)$ data and weighted residual error of the 6 results, and calculate the mean value and RSD. The determination results are shown in Table 8.

The injection speed has little effect on the particle size measurement results. When the injection speed is $40 \%$, the RSD value and average residual of the six measurements are the minimum, so the injection speed was set as $40 \%$.

Table 6. Investigation results with different ultrasonic time $(n=6)$.

\begin{tabular}{|c|c|c|c|c|}
\hline Ultrasonic time $/ \mathrm{s}$ & $d(0.1) / \mu \mathrm{m}$ & $d(0.5) / \mu \mathrm{m}$ & $d(0.9) / \mu \mathrm{m}$ & Mean residual $/ \%$ \\
\hline 30 & $0.825(2.16)$ & $2.72(5.75)$ & $7.07(5.45)$ & 5.56 \\
\hline 60 & $0.813(1.16)$ & $2.66(2.75)$ & $7.88(3.08)$ & 1.12 \\
\hline 120 & $0.821(1.73)$ & $2.71(3.07)$ & $7.92(3.61)$ & 1.23 \\
\hline
\end{tabular}

Table 7. Investigation results with different stirring speed $(n=6)$.

\begin{tabular}{|c|c|c|c|c|}
\hline Mixing speeds/rpm & $d(0.1) / \mu \mathrm{m}$ & $d(0.5) / \mu \mathrm{m}$ & $d(0.9) / \mu \mathrm{m}$ & Mean residual $/ \%$ \\
\hline 1200 & $0.814(2.32)$ & $2.78(3.43)$ & $6.34(4.65)$ & 4.23 \\
\hline 1400 & $0.827(2.10)$ & $2.81(4.36)$ & $6.71(5.46)$ & 5.33 \\
\hline 1600 & $0.820(0.93)$ & $2.85(1.04)$ & $7.83(1.32)$ & 0.98 \\
\hline 2000 & $0.815(1.25)$ & $2.63(2.21)$ & $8.32(3.95)$ & 1.71 \\
\hline 2500 & $0.828(1.21)$ & $2.73(1.43)$ & $8.56(2.35)$ & 1.43 \\
\hline
\end{tabular}

Table 8. Investigation results with different injection speed $(n=6)$.

\begin{tabular}{|c|c|c|c|c|}
\hline Injection speeds $/ \mathrm{rpm}$ & $d(0.1) / \mu \mathrm{m}$ & $d(0.5) / \mu \mathrm{m}$ & $d(0.9) / \mu \mathrm{m}$ & Mean residual $/ \%$ \\
\hline 35 & $0.815(1.10)$ & $2.75(1.36)$ & $7.91(1.46)$ & 1.56 \\
\hline 40 & $0.827(0.93)$ & $2.72(1.04)$ & $7.85(1.32)$ & 0.68 \\
\hline 45 & $0.825(1.25)$ & $2.72(2.21)$ & $8.32(3.95)$ & 0.97 \\
\hline 50 & $0.819(1.21)$ & $2.83(1.43)$ & $8.57(2.35)$ & 2.21 \\
\hline
\end{tabular}


Table 9. Results of particle size $(n=6)$.

\begin{tabular}{|c|c|c|c|}
\hline Batch No. & $d(0.1) / \mu \mathrm{m}$ & $d(0.5) / \mu \mathrm{m}$ & $d(0.9) / \mu \mathrm{m}$ \\
\hline 20191001 & $0.808(1.53)$ & $2.77(2.51)$ & $8.50(2.32)$ \\
\hline 20191002 & $0.825(0.96)$ & $2.80(2.54)$ & $8.36(1.39)$ \\
\hline 20191003 & $0.825(1.04)$ & $2.79(2.58)$ & $8.45(0.91)$ \\
\hline
\end{tabular}

\section{Validation of the method \\ Precision test}

Samples were prepared as outlined in subsection 2.1 and the parameters were set as previously determined. For each sample PSD measurements were taken 6 times., The RSD of the results $d(0.1)$ is $1.37 \%$, the RSD of $d(0.5)$ is $1.60 \%$, and the RSD of $d(0.9)$ is $1.05 \%$, indicating that the method has good precision.

\section{Repeatability test}

For this test, 6 replicate samples were prepared and their PSD measurements were determined. The results show that the RSD of $\mathrm{d}(0.1)$ is $1.61 \%$, the RSD of $d(0.5)$ is $1.54 \%$, and the RSD of $d(0.9)$ is $1.48 \%$, implying that the method has good repeatability.

\section{Determination of tadalafil drug substance particle size}

3 batches of tadalafil drug substance were prepared and the particle size distribution was determined. The mean value and RSD of $d(0.1), d(0.5)$, $d(0.9)$ of each batch of samples, the results are shown in Table 9.

\section{Comparison with electron microscopic observations}

In order to further verify the accuracy of the results of this method, we also performed electron microscopy and the morphology of tadalafil drug substance (14) as shown in Figure 1. As shown in the figure, the particle size of tadalafil drug substance is almost always below $10 \mu \mathrm{m}$, which is basically consistent with the particle size distribution results determined by laser particle size meter. Furthermore, this correlation strongly indicates that the results of this method employed using the laser particle analyzer are accurate.

Figure 1. SEM picture of tadalfil drug substance.

\section{DISCUSSION AND CONCLUSION}

In this study, the particle size of tadalafil drug substance was determined by wet method of laser scattering. Tadalafil is almost insoluble in water, and it is not easy to disperse evenly in water. At the same time, the particles are very fine, the density is small, the weight is light, and it is easy to generate static electricity. It was found adding an appropriate amount of surface-active agent can make it evenly dispersed in water. Tween 80 is commonly used as a surfactant that maximizes the wetting of the sample and destroys the adhesion between the molecules. At the same time, after reviewing a large amount of literature (15-18), we found that Tween was often used as the surfactant when the Malvern laser particle size analyzer was used to detect the particle size of API by the wet method.

In this experiment, through the investigation of the detection conditions such as dispersion pressure, ultrasonic speed, ultrasonic time, and stirring speed, the appropriate parameters were evaluated and selected. The precision and repeatability of the method were verified and the results showed that the method was simple, precise and reproducible, and could accurately and scientifically determine the particle size distribution of tadalafil drug substance. Comparatively the results were basically consistent with those by scanning electron microscopy (SEM) (19-20).

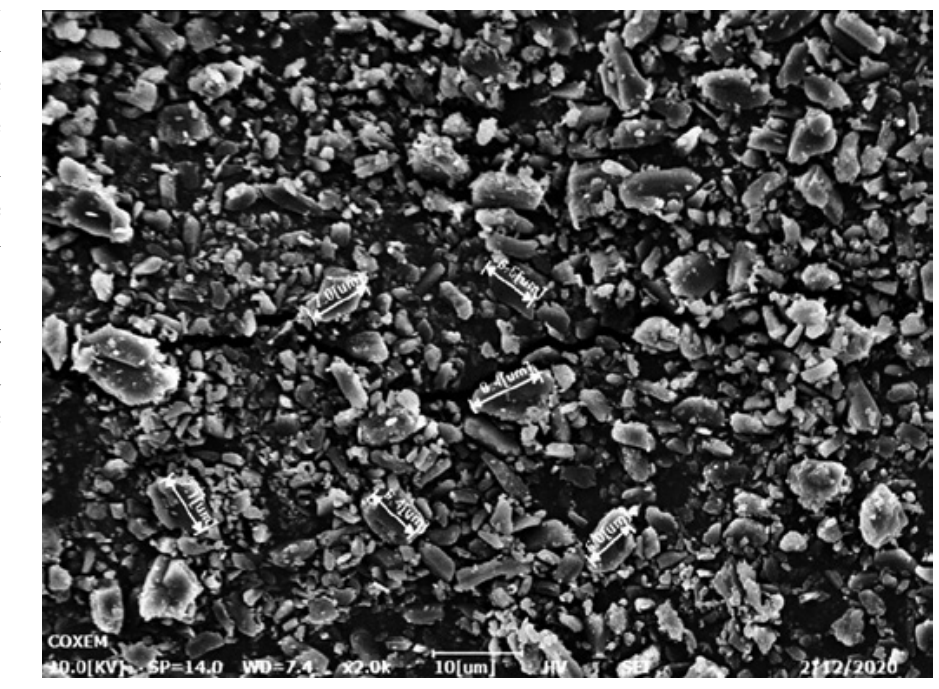


The method can be used for the determination of tadalafil particle size. Meanwhile, it provides an experimental basis and test methods for the quality control of tadalafil drug substance and has been successfully applied in the actual development and production of tadalafil drug products.

\section{Conflict of interest}

The authors declare no conflicts of interest.

\section{REFERENCES}

1. Gong B.B, Ma M., Xie W.J., Yang X.R, Huang Y.M., et al.: Int. Urol. Nephrol. 49, 1731 (2017).

2. Washington S.L., Shindel A.W.: Drug Des. Devel. Ther. 4, 159 (2010).

3. Falk J.A., Philip K.J., Schwarz E.R.: Vasc. Health Risk Manag. 6, 273 (2010).

4. Simoes S., Sousa A., Figueiredo M.: Int. J. Pharm. 127, 283 (1996).

5. Swaminathan V., Kildsig D.O.: Drug Dev. Ind. Pharm. 28, 41 (2002).

6. Shiraishi T., Sano A., Kondo S., Yuasa H., Kanaya Y.: Chem. Pharm. Bull. 43, 654 (1995).

7. Yajima T., Itai S., Hayashi H., Takayama K., Nagai T.: Chem. Pharm. Bull. 44, 1056 (1996).
8. Yao T., Yamada M., Yamahara H.., Yoshida M.: Chem. Pharm. Bull. 46, 826 (1998).

9. Silva A.F., Burggraeve A., Denon Q., Van der Meeren P., Sandler N., et al.: Eur. J. Pharm. Biopharm. 85, 1006 (2013).

10. Shekunov B.Y., Chattopadhyay P., Tong H.H., Chow A.H.: Pharm. Res. 24, 203 (2007).

11. Song Y., Xun M., Feng Z., et al.: Central Pharmacy 2020, 474 (2020).

12. Xun M, Feng Z., Zhang G., et al.: Pharm. Res. 39,213 (2020).

13. Geng Z., Jiang Y., Li Y., et al.: J. China Pharmaceutical Industry 48, 716 (2017).

14. Ma Z., Merkus H.G., De Smet J.G.A.E., Heffels C., Scarlett B.: Powder Technol. 11166 (2000).

15. Shen D., Zeng L., Xu J., et al.: Chinese Journal of Antibiotics 44, 946 (2019).

16. Wang H., Liao M., Guan Q.: China Pharmaceutical Industry 28, 29 (2019).

17. Zhang C., Hu R., Yang X., et al.: J. Southwest University for Nationalities (Natural Science Edition) 34, 507 (2008).

18. Zou W., Wang Y., Yin L., et al.: Pharm. Res. 37, 93 (2018).

19. Greaves D., Boxall J., Mulligan J., Montesi A., Creek J., et al.: Chem. Eng. Sci. 63, 5410 (2008).

20. Sandler N.: Int. J. Pharm. 417, 227 (2011). 